\title{
Structured Grammatical Evolution for Glucose Prediction in Diabetic Patients
}

\author{
Nuno Lourenço \\ CISUC, Department of Informatics Engineering, \\ University of Coimbra \\ Coimbra, Portugal \\ naml@dei.uc.pt \\ J. Ignacio Hidalgo \\ Universidad Complutense de Madrid \\ Madrid, Spain \\ hidalgo@dacya.ucm.es
}

\author{
J. Manuel Colmenar \\ Universidad Rey Juan Carlos \\ Móstoles, Spain \\ josemanuel.colmenar@urjc.es
}

\author{
Óscar Garnica \\ Universidad Complutense de Madrid \\ Madrid, Spain \\ ogarnica@ucm.es
}

\begin{abstract}
Structured grammatical evolution is a recent grammar-based genetic programming variant that tackles the main drawbacks of Grammatical Evolution, by relying on a one-to-one mapping between each gene and a non-terminal symbol of the grammar. It was applied, with success, in previous works with a set of classical benchmarks problems. However, assessing performance on hard real-world problems is still missing. In this paper, we fill in this gap, by analyzing the performance of SGE when generating predictive models for the glucose levels of diabetic patients. Our algorithm uses features that take into account the past glucose values, insulin injections, and the amount of carbohydrate ingested by a patient. The results show that SGE can evolve models that can predict the glucose more accurately when compared with previous grammarbased approaches used for the same problem. Additionally, we also show that the models tend to be more robust, since the behavior in the training and test data is very similar, with a small variance.
\end{abstract}

\section{CCS CONCEPTS}

- Computing methodologies $\rightarrow$ Artificial intelligence; • Applied computing $\rightarrow$ Health informatics; • Theory of computation $\rightarrow$ Algorithm design techniques;

\section{KEYWORDS}

Grammatical Evolution, Structured Grammatical Evolution, Performance.

\section{ACM Reference Format:}

Nuno Lourenço, J. Manuel Colmenar, J. Ignacio Hidalgo, and Óscar Garnica. 2019. Structured Grammatical Evolution for Glucose Prediction in Diabetic Patients. In Genetic and Evolutionary Computation Conference (GECCO '19), fuly 13-17, 2019, Prague, Czech Republic. ACM, New York, NY, USA, 9 pages. https://doi.org/10.1145/3321707.3321782

Permission to make digital or hard copies of all or part of this work for personal or classroom use is granted without fee provided that copies are not made or distributed for profit or commercial advantage and that copies bear this notice and the full citation on the first page. Copyrights for components of this work owned by others than ACM must be honored. Abstracting with credit is permitted. To copy otherwise, or republish, to post on servers or to redistribute to lists, requires prior specific permission and/or a fee. Request permissions from permissions@acm.org.

GECCO '19, July 13-17, 2019, Prague, Czech Republic

(C) 2019 Association for Computing Machinery.

ACM ISBN 978-1-4503-6111-8/19/07 . \$ \$15.00

https://doi.org/10.1145/3321707.3321782

\section{INTRODUCTION}

Diabetes is a metabolic disorder that affects an increasing number of people all around the world. According to the International Diabetes Federation, 425 million people suffer from this illness and this number is expected to grow to 629 million by the year 2045 [6]. The global economic costs of diabetes, as of 2017, was estimated to be US $\$ 727$ billion. The disease results from the insufficient production of insulin by the pancreas or by a problem in the body cells, which do not respond appropriately to the insulin that the pancreas produces. In both cases, the glucose (sugar) levels in the bloodstream rise, which leads to life-threatening complications such as brain or kidney injuries. Additionally, there are also risks associated with hypoglycemia, which happens when the levels of glucose drop drastically.

Patients need to oversee the glucose levels, trying to keep them between a range of acceptable values: [70-180] mg/dl. Before eating, a diabetic patient needs to estimate the units of insulin that need to be injected, so that after the meal the glucose levels are in a healthy range. This estimation is usually made taking into account the current levels of glucose (measured before the eating) and the amounts of food that are going to be ingested, measured in carbohydrate rations. This process is error-prone since there are no clear processes defined and it involves several estimations. However, in recent years, scientific progress in devices and algorithms allowed for the automation of some parts of this process. Nowadays many monitoring systems can continuously measure the levels of glucose, which can alleviate the painful process of estimating this value by the patient. The joint work of these devices and algorithms to generate prediction models is the key point to increase the quality of life of diabetic patients.

In this paper, we use Structured Grammatical Evolution (SGE) [13] to generate prediction models taking into account the past glucose values, insulin injections, and the amount of carbohydrate ingested. Our goal is to assess the performance of SGE in a hard realworld problem. As a baseline comparison, we rely on Grammatical Evolution (GE) [16], which has been used to tackle the same problem with success [7].

The results show that SGE can evolve models that predict the glucose accurately. Additionally, we also show that the SGE models tend to be more robust than GE ones since the behavior in the training and test data is very similar, but with a small variance. 
These results show that SGE can be used in the construction of automated methods that can increase the quality of life of the millions of people that have diabetes.

The remainder of the papers is as follows: in Section 2 we introduce the two Grammar-Based Genetic Programming approaches used in our work. In Section 3 we detail the problem and the data we have used. Section 4 details our experimental setup, and reports the results. Finally, in Section 5 we gather the main conclusions and points towards future work.

\section{GENETIC PROGRAMMING AND GRAMMARS}

Genetic Programming (GP) [10] is an evolutionary methodology that considers the evolution of programs represented as trees with the aim of tackling an optimization problem. Given that a program requires a set of syntactical rules to, at least, compile and execute, many proposals around using grammars to help GP in the optimization process came up soon. However, as stated in [14], there is a separation between Grammar Guided Genetic Programming (GGGP) and Grammatical Evolution (GE). On the one hand, GGGP methods use grammars as components that collaborate in the management of the derivation trees. For instance, in CFG-GP [18], a context-free grammar is used to generate the initial population of the GP algorithm. This way, a set of correct derivation trees is generated, and their correctness among the evolutionary process is maintained by the operators. On the other hand, GE [16] uses the grammar to obtain a derivation tree from the individuals of the population, which are encoded as chromosomes using either binary of integer numbers.

Therefore, the behavior and performance of GGGP and GE methods are different, being GE one of the most widely applied variants of GP [1], [3], [4], [9], [15], [17]. In this paper, we show how Structured Grammatical Evolution (SGE) [13], which is a recent proposal from the GE family, overtakes GE in a real-world problem, which is the prediction of future glucose values in diabetic patients. In particular, we consider the implementation of GE that was already used in this very same problem [7], [8], which is the Java for Evolutionary COmputation library (JECO), freely available in GitHub ${ }^{1}$.

Structured Grammatical Evolution (SGE) [13] is a recent variant of GE that tackles its main drawbacks. In particular, SGE relies on a one-to-one mapping between each gene and a non-terminal symbol of the grammar. This genotypic organization establishes that a change in one of the genes does not affect the derivation options of other non-terminals, thus narrowing the changes that can occur at the phenotypic level (i.e., the derivation tree). This way the locality of the representation is increased. Moreover, the values inside each list are limited to the number of expansion options of the corresponding non-terminal. Consequently, the modulus operator is not needed, which reduces the redundancy introduced by it.

In order to illustrate the decoding process of SGE, we describe it with a small example. Figure 1 shows a context-free grammar for polynomial expressions with a set of four non-terminal symbols, denoted as $N$, eight terminal symbols, denoted as $T$, the starting symbol $S$ and four production rules denoted as $P$, and corresponding to each one of the four non-terminal symbols.

\footnotetext{
${ }^{1}$ https://github.com/ABSysGroup/JECO
}

$$
\begin{aligned}
& N=\{<\text { start }>,<\operatorname{expr}\rangle,<\text { term }>,<o p>\} \\
& T=\left\{+,-, /, *,(,), x_{1}, 0.5\right\} \\
& S=\{<\text { start }>\} \\
& \text { And the production set } \mathrm{P} \text { is: } \\
& <\text { start }>::=<\operatorname{expr}><\text { op }><\operatorname{expr}>(0) \\
& \text { | <expr }> \\
& <\text { expr }>::=<\text { value }><\text { op }><\text { value }>(0) \\
& \text { | }(<\text { value }><\text { op }><\text { value }>\langle 1) \\
& <\mathrm{op}>::=+(0) \\
& -(1) \\
& \text { | / (2) } \\
& \text { | * (3) } \\
& <\text { value }>::=x_{1}(0) \\
& \text { | } 0.5(1)
\end{aligned}
$$

Figure 1: Example of a Context-Free-Grammar in the Backus-Naur form. $N$ is the set of non-terminal symbols, $T$ is the set of terminal symbols and $S$ is the grammar's axiom. Adapted from [11].

Table 1: Mapping procedure that converts an individual (first row, right column) into a polynomial expression using SGE. Each row represents a derivation step using the grammar in Figure 1. Each gene has the list of integers needed for expanding $<$ start $>$, $<$ expr $>$, $<$ op $>$ and $<$ value $>$, respectively. Adapted from [11].

\begin{tabular}{l|l} 
Derivation step & Integers left \\
\hline$<$ start $>$ & {$[[0],[1,0],[2,0,3],[1,1,0,0]]$} \\
$<$ expr $><$ op $><$ expr $>$ & {$[[],[1,0],[2,0,3],[1,1,0,0]]$} \\
$(<$ value $><$ op $><$ value $>)<$ op $><$ expr $>$ & {$[[],[0],[2,0,3],[1,1,0,0]]$} \\
$(0.5<$ op $><$ value $>)<$ op $><$ expr $>$ & {$[[],[0],[2,0,3],[1,0,0]]$} \\
$(0.5 /<$ value $>)<$ op $><$ expr $>$ & {$[[],[0],[0,3],[1,0,0]]$} \\
$(0.5 / 1)<$ op $><$ expr $>$ & {$[[],[0],[0,3],[0,1]]$} \\
$(0.5 / 1)+<\operatorname{expr}>$ & {$[[],[0],[3],[0,1]]$} \\
$(0.5 / 1)+<$ value $><$ op $><$ value $>$ & {$[[],[],[3],[0,0]]$} \\
$(0.5 / 1)+x_{1}<$ op $><$ value $>$ & {$[[],[],[3],[0]]$} \\
$(0.5 / 1)+x_{1} *<$ value $>$ & {$[[],[],[],[0]]$} \\
$(0.5 / 1)+x_{1} * x_{1}$ & {$[[],[],[],[]]$}
\end{tabular}

Since the grammar has four non-terminals, the genotype of each SGE individual will have four genes. Each one of these genes will be linked to a specific non-terminal. To calculate the length of the lists inside each gene, the algorithm computes the maximum number of derivation options for each non-terminal. Table 1 shows an example of the genotype of an individual created using the 
grammar of Figure 1, and how we derive it into a mathematical expression.

In this work, we consider the most recent SGE version, freely available on GitHub ${ }^{2}$. The main feature of this version is that, instead of computing and generating the maximum number of derivations for each of the non-terminal symbols, a variable length representation is used. As such, there is no need to create intermediate symbols to deal with recursive rules. However, there is the need to limit the size of the genotype. This is achieved by specifying the maximum depth that the derivation tree can have. This means that the trees will grow until a certain limit. Notice that in the case of GE, this control of the depth of the derivation tree is made with the maximum value of wraps.

\section{GLUCOSE PREDICTION: A REAL WORLD PROBLEM}

In the past, GE has been successfully used to build models for glucose prediction in diabetic patients [7], [8]. Besides, in [12], Lourenço et al. showed that SGE has a better performance than GE on several different problems. These works motivated us to compare the performance of SGE with GE in the problem of glucose modeling, a real-world problem that will be described in this section.

Diabetes patients need to decide the amount of insulin they have to inject themselves in order to process the food and maintain the blood glucose values in an acceptable range. For this decision, they use all the information available, which is usually the current and past glucose values, the number of carbohydrates in the intakes and the past values of insulin. In other words, the objective is to predict the value of blood glucose concentration based upon previous blood glucose values and the injection of some units of insulin with the eating of a number of carbohydrates. We consider a forecasting window of two hours $(t+120)$. The data are available as a timeseries with observations in a regular interval of five minutes. We transform the problem into a regression problem in the form:

$$
\begin{aligned}
\hat{G}_{t+120}= & f_{t+120}\left(\left(G_{t+i}, i \in(-240 \ldots 0)\right),\right. \\
& \left(I_{t+i}, C_{t+i}, i \in(-240 \ldots+120)\right)
\end{aligned}
$$

where $\hat{G}$ is the forecast for blood glucose, $G$ is the time series of measured blood glucose concentration values, $I$ is the time series of insulin inputs, and $C$ is the number of carbohydrate inputs as estimated by the patients. We consider that at each time point, $t$, data from up to four hours before are available for prediction $G_{t+i}, i \in(-240 \ldots 0), I_{t+i}, C_{t+i}, i \in(-240 \ldots+120)$. We use the same data and the same preprocessing used in [7], where ten patients $(n=10)$ were selected based on conditions of good glucose control for an observational study. Data from patients were acquired over multiple weeks using Medtronic continuous glucose monitors (CGMs) stored in five-minute intervals. Each entry records the amount of carbohydrate intake as estimated by the patients, the amount of insulin injected, and the blood glucose value for a time instant. Table 2 shows some statistics about the glucose levels of

\footnotetext{
${ }^{2}$ https://github.com/nunolourenco/dsge
}

the data acquired from the patients as well as the number of data points for each patient (\# Points column).

\section{EXPERIMENTAL STUDY}

\subsection{Experimental Setup}

In order to have a fair comparison between GE and SGE, we use the same numerical parameters for both algorithms whenever is possible, whose values were selected after preliminary experimentation. Both algorithms have a population of 200 individuals that are evaluated throughout 250 generations. We allow $10 \%$ of the best individuals to survive untouched and pass along to the next generation without any changes. The probabilities of applying the variation operators are the same for both algorithms. The main differences are concerned with parameters related to the genotypic representation. For SGE we have a genotype that allows derivation trees with depth varying between 4 and 17, and for GE we defined a genotype of size 200. Each algorithm is executed 30 times in order to allow for statistical analysis. Table 3 summarizes the parameters of each algorithm.

\subsection{Grammar}

In this section, we present the grammar used by GE and SGE. The grammar takes into account the nature of the problem, i.e., glucose prediction, and relies on non-terminals that represent variables related to the domain-specific knowledge. We know that our final models have to be a combination of variables that measure the current levels of glucose, the estimation of the carbohydrate and the amount of insulin. This information is represented in the grammar through the use of the non-terminals $<$ vargl $>$, <varch $>$ and $<$ varins $>$, respectively. The goal of the grammar is to generate polynomial expressions that combine the input variables with mathematical operators and constants. There are a total of 39 input variables which are represented in the grammar by $X[1], X[2], \ldots, X[39]$. One of the advantages of GE is that we can select the variables (i.e., the features) through the grammar. For instance, Figure 2 presents a set of productions where some of the variables are omitted while others are combined by addition. We selected this grammar after considering the possible redundancy of information if we include all the variables.

\subsection{Metrics}

\section{Root Mean Square Error}

The evolutionary process of both approaches is guided by the minimization of the Root Mean Squared Error (RMSE) between the original data and the predictions by the models being evolved, which is defined as:

$$
\mathrm{RMSE}=\sqrt{\frac{1}{T} \sum_{i=1}^{T}\left(\hat{y}_{i}-y_{i}\right)^{2}}
$$

where $T$ is the number of data points, $\hat{y}_{i}$ is the predicted value for sample $i$, and $y_{i}$ is the reference value for sample $i$. 
Table 2: Descriptive statistics for the data sets for all ten patients. Values in range $\mathbf{1 8 0 - 2 5 0}$ are $(100 \%-$ Time glucose $<70-$ Time glucose $_{>250}-$ Time in target $_{70-180}$ ).

\begin{tabular}{ccccccc}
\hline Patient & $\begin{array}{c}\text { Average } \\
(\mathrm{mg} / \mathrm{dl})\end{array}$ & $\begin{array}{c}\text { Std. Deviation } \\
(\mathrm{mg} / \mathrm{dl})\end{array}$ & $\begin{array}{c}\text { Time glucose } \\
<70 \mathrm{mg} / \mathrm{dl}\end{array}$ & $\begin{array}{c}\text { Time glucose } \\
>250 \mathrm{mg} / \mathrm{dl}\end{array}$ & $\begin{array}{c}\text { Time in target } \\
{[70-180] \mathrm{mg} / \mathrm{dl}}\end{array}$ & \# Points \\
\hline 1 & 157.67 & 62.25 & $4.48 \%$ & $7.31 \%$ & $37.57 \%$ & 4018 \\
2 & 145.44 & 64.02 & $8.33 \%$ & $6.18 \%$ & $41.83 \%$ & 23534 \\
3 & 143.46 & 45.45 & $2.14 \%$ & $2.26 \%$ & $49.18 \%$ & 23821 \\
4 & 150.47 & 56.70 & $4.12 \%$ & $5.14 \%$ & $41.29 \%$ & 20090 \\
5 & 139.17 & 67.93 & $14.17 \%$ & $7.10 \%$ & $41.89 \%$ & 8036 \\
6 & 142.58 & 60.08 & $10.08 \%$ & $4.44 \%$ & $41.21 \%$ & 12628 \\
7 & 176.33 & 68.35 & $3.65 \%$ & $14.28 \%$ & $27.07 \%$ & 6888 \\
8 & 135.34 & 46.11 & $4.85 \%$ & $1.84 \%$ & $54.06 \%$ & 6027 \\
9 & 146.82 & 59.82 & $7.54 \%$ & $5.20 \%$ & $39.49 \%$ & 5740 \\
10 & 166.13 & 86.12 & $9.33 \%$ & $16.16 \%$ & $35.69 \%$ & 4305 \\
\hline
\end{tabular}

Table 3: Parameters used in the experimental analysis for each method.

\begin{tabular}{lcc}
\hline & \multicolumn{2}{c}{ Value } \\
\hline Parameter & SGE & GE \\
\hline Number of runs & \multicolumn{2}{c}{200} \\
Population Size & \multicolumn{2}{c}{250} \\
Generations & \multicolumn{2}{c}{ Tournament with size 3 } \\
Selection Method & \multicolumn{2}{c}{$10 \%$} \\
Elitism & \multicolumn{2}{c}{0.9} \\
Crossover Rate & 0.05 & 0.05 \\
Mutation Rate & Structured & Single-Point \\
Crossover & Pointwise & Pointwise length) \\
& (per codon) & (per codon) \\
Mutation & Uniform & Uniform \\
Crossover Node Selection & Random & Random \\
Initialisation & 4 & - \\
Min. Initialisation Depth & 6 & - \\
Max. Initialisation Depth & 17 & - \\
Max. Tree Depth & - & 200 \\
Initial Genotype Length & - & None \\
Wrapping &
\end{tabular}

\section{Clarke Error Grid Analysis}

Clarke Error Grid [2] is usually constructed as a tool for quantifying the clinical significance of a prediction or measurement of glucose values. It is used to show the differences between the predicted values and the reference values by representing in a Cartesian diagram the points formed by the pair (predicted values, actual value). In this way a point indicates that the actual value was $x$ and the predicted value was $y$, being $y=x$ the perfect prediction. After plotting all these pairs, the graphic is divided into a grid of zones, which indicates the severity of an erroneous prediction [7]. There are five zones delimited in the graph (A to E), with the following meanings:

- Zones A and B: values on Zone A represents the glucose values that deviate from the reference values by $20 \%$ or less and those that are in the hypoglycemic range $(<70 \mathrm{mg} / \mathrm{dl})$,

$$
\begin{aligned}
&<\text { start }>::=<\text { expr }> \\
&<\operatorname{expr}>::=(<\text { expr }><\text { op }><\text { expr }>) \\
& \mid(<\text { cte }><\text { op }><\text { expr }>) \\
& \mid<\text { var }> \\
&<\text { op }>::=+|-| * \\
&<\text { var }>::=<\text { varch }>\mid<\text { varins }>\mid<\text { vargl }> \\
&<\text { vargl }>::=x[2]|x[4]| x[7] \\
&<\text { varch }>::=x[9] \mid(x[10]+x[11]) \\
& \\
&|(x[12]+x[13])|(x[14]+x[15]) \\
&|(x[16]+x[17])|(x[18]+x[19]) \\
&|(x[20]+x[21])|(x[22]+x[23]) \\
&<\text { varins }>::=x[24] \mid(x[25]+x[26]) \\
&|(x[27]+x[28])|(x[29]+x[30]) \\
&|(x[31]+x[32])|(x[33]+x[34]) \\
&|(x[35]+x[36])|(x[37]+x[38]) \\
&<\text { cte }>::=< \text { factor }>*<\text { digit }> \\
&<\text { factor }>::=0.1|0.01| 0.001|0.0001| 1|10| 100 \\
&<\text { digit }>::=0|1| 2|3| 4|5| 6|7| 8|9| 10
\end{aligned}
$$

Figure 2: Grammar used for both GE and SGE experiments.

not only the predicted value but also the reference value. Predicted values on zone B deviate from the reference values by more than $20 \%$ although the clinical treatment will be correct with a high probability. The values that fall within zones A and B are clinically exact and/or acceptable, and thus the clinical treatment will be correct. We will treat those classes as a combined category in the analysis of our experimental results.

- Zone C: The values in this zone could be dangerous in some situations. The goal is to minimize predictions in this category. 


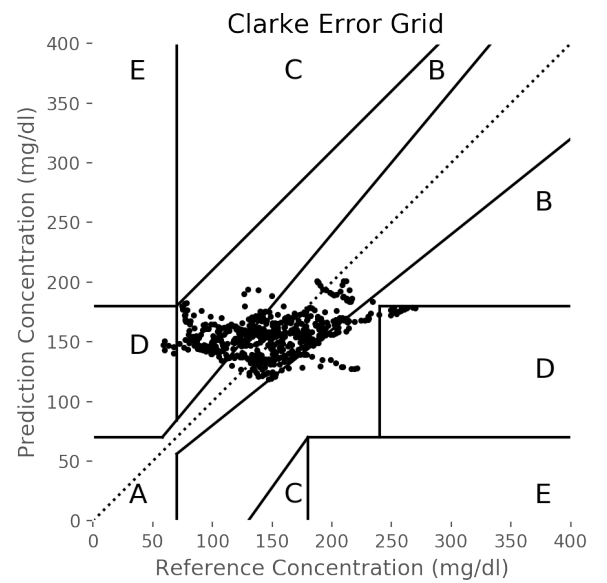

Figure 3: Example of the Clarke Error Grid for Patient 1, considering 120 minutes predictions.

Table 4: Mean Best Fitness and Standard Deviation results obtained during training. Results are average of 30 runs. Bold values indicate that there are statistical significant differences. The $*$ are concerned with the effect size (see text)

\begin{tabular}{ccc}
\hline Patient & GE & SGE \\
\hline 1 & $49.94( \pm 2.43)$ & $\mathbf{4 8 . 5 4}( \pm \mathbf{0 . 2 2})^{* * *}$ \\
2 & $52.81( \pm 4.73)$ & $\mathbf{5 0 . 1 0}( \pm \mathbf{0 . 4 7})^{* * *}$ \\
3 & $38.08( \pm 2.01)$ & $\mathbf{3 7 . 0 1}( \pm \mathbf{0 . 2 7})^{* *}$ \\
4 & $48.90( \pm 2.48)$ & $\mathbf{4 7 . 6 4}( \pm \mathbf{0 . 2 3})^{* * *}$ \\
5 & $58.63( \pm 2.92)$ & $\mathbf{5 6 . 7 1 (}( \pm \mathbf{0 . 4 2})^{* * *}$ \\
6 & $52.57( \pm 3.59)$ & $\mathbf{5 0 . 7 4}( \pm \mathbf{0 . 3 7})^{* * *}$ \\
7 & $65.26( \pm 5.13)$ & $\mathbf{6 2 . 3 6}( \pm \mathbf{0 . 3 6})^{* * *}$ \\
8 & $44.45( \pm 3.09)$ & $43.16( \pm 0.18)$ \\
9 & $49.79( \pm 2.65)$ & $48.74( \pm 0.28)$ \\
10 & $75.24( \pm 5.07)$ & $\mathbf{7 2 . 6 8 ( \pm \mathbf { 0 . 5 2 }})^{* *}$ \\
\hline
\end{tabular}

- Zones D-E: The values included in those areas are potentially dangerous since the prediction is far from being acceptable and the indicated treatment will be different from the correct. Again, the goal is to minimize predictions in this category.

Figure 3 shows an example of the Clarke Error Grid for Patient 1- Horizon 120 minutes predictions.

\subsection{Results}

\section{Training Results}

The experiments in this section aim to gain insight on the ability of both GE and SGE to discover models that can accurately predict the glucose values in a time window of 120 minutes. The results are presented in terms of mean best fitness averaged over 30 runs, complemented with the standard deviation values. To compare the approaches we use the Mann-Whitney test due to the characteristics of the data and the fact that the initial populations are different. We use a significance level $\alpha=0.05$. When statistical differences are found, we compute the effect size to assess how meaningful
Table 5: Mean Best Fitness and Standard Deviation results obtained during test. Results are average of 30 runs. Bold values indicate that there are statistical significant differences. The $*$ are concerned with the effect size (see text)

\begin{tabular}{|c|c|c|}
\hline Patient & GE & SGE \\
\hline 1 & $50.58( \pm 2.32)$ & $48.00( \pm 0.51)^{* * *}$ \\
\hline 2 & $52.71( \pm 4.69)$ & $49.44( \pm 0.48)^{* * *}$ \\
\hline 3 & $38.12( \pm 1.95)$ & $36.85( \pm 0.30)^{* * *}$ \\
\hline 4 & $48.87( \pm 2.41)$ & $47.47( \pm 0.23)^{* * *}$ \\
\hline 5 & $59.80( \pm 2.80)$ & $57.49( \pm 0.51)^{* * *}$ \\
\hline 6 & $52.87( \pm 3.37)$ & $50.58( \pm 0.38)^{* * *}$ \\
\hline 7 & $55.05( \pm 4.56)$ & $51.84( \pm 0.64)^{* * *}$ \\
\hline 8 & $44.77( \pm 3.20)$ & $42.72( \pm 0.29)^{* * *}$ \\
\hline 9 & $50.44( \pm 2.64)$ & $48.72( \pm 0.57)^{* * *}$ \\
\hline 10 & $76.00( \pm 4.89)$ & $72.27( \pm 1.02)^{* * *}$ \\
\hline
\end{tabular}

the differences are. The effect size is denoted by the number of $*$ signals, where $*, * *$ and $* * *$ correspond respectively to low $(0.1 \leq$ $\mathrm{r}<0.3)$, medium $(0.3 \leq \mathrm{r}<0.5)$ and large $(\mathrm{r} \geq 0.5)$ effect sizes [5].

Table 4 presents the training results for both GE and SGE for all the patients. The results are averages of 30 runs in 8 -fold crossvalidation. A brief perusal of the results shows that SGE obtains smaller error values when compared with GE. This result is consistent across all the patients considered in the study. Moreover, it is possible to see that the standard deviation values for SGE are also smaller. This is a significant result, which indicates that SGE is robust since it can find better models than GE and the difference, in terms of RMSE, between separate runs is small.

Concerning statistical comparisons, we find that in 8 out of 10 patients the differences between SGE and GE are significant, with SGE being better. For patients 8 and 9 we do not find any meaningful differences. However, the error presented by SGE is smaller. To complement the results, we compute the effect size of the differences. The effect size is large in 6 out of 8 and medium for the remaining two.

\section{Testing Results}

When creating predictive models, the results on testing (i.e., unseen) data are more important than the results on training. It is in this phase that we are able to estimate how well the models have been trained and can accurately predict the glucose evolution of patients.

Table 5 shows the RMSE obtained in the test phase. The results are averages of 30 runs. This means that we take each one of the models obtained for each run in the training and apply it to the test data. The results show the same trend obtained during the training phase. The models evolved by SGE show a smaller error when compared with GE. The SGE models also show a smaller standard deviation. Figure 4 shows the results for GE and SGE, and for training and testing over all the patients. These plots confirm the good performance of SGE and also show that none of these approaches overfit. It is important to realize that the variance of GE between the training and test phases is not consistent. For example, in Patient 9 it is possible to see that the variance in the testing phase is much larger than in the training phase. This is an indication that the models discovered by GE tend to be less robust. 
Concerning statistical comparisons, we find that the differences between SGE and GE are significant for all the patients considered. The effect size of the differences is important for all the comparisons.

As mentioned previously, it is important to use a metric to evaluate the performance of the models that is recognized by the clinical staff. In Figure 5 we present the results using the Clarke Error Grid described above. We present the results in terms of percentage of total points. A brief perusal of the table shows that when we consider only zones A and B together, there are not many differences between GE and SGE. However, looking at the zones C, D, and $\mathrm{E}$, which represent predictions that could lead to a dangerous treatment, it is possible to see that, in general, GE shows a higher percentage of points in these zones. Note that for the most dangerous one, the zone E, the percentage of points is $1.7 \%$ for GE and less than $1.4 \%$ for SGE. This is an indication that the models evolved by SGE tend to be more robust and can predict the glucose levels accurately.

\section{CONCLUSIONS AND FUTURE WORK}

In this paper, we study the performance of the Structured Grammatical Evolution (SGE) method on a real-world problem consisting on the generation of model expressions to predict the blood glucose value of diabetic patients using a horizon of 120 minutes. SGE is a form of Grammatical Evolution (GE) which represents the individuals as chromosomes where each gene is formed by a list of values corresponding to the productions of each non-terminal symbol. This way, the decoding process is different to GE and can increase the locality of the genetic operators. Therefore, the behavior of SGE is compared with a standard GE implementation. To this aim, we consider data from ten real patients with different shapes and trends in their glucose values. The dataset is divided into training and test sets, and both GE and SGE are configured to spend the same computational effort. The experimental results show two different metrics. On the one hand, we compute the error using the root mean square error (RMSE). On the other hand, we analyze a metric that is particularly important to the study of diabetes, the Clarke Error Grid. In both metrics, SGE reaches better results than GE, obtaining models that are also more robust concerning the relationship between the training and the test results. Our statistical analysis also confirms that the differences between GE and SGE are significant in all the patients.

As future work, we will study more prediction horizons, as well as the hybridization with other techniques like classification algorithms in order to manage different models for data with different behavior.

\section{ACKNOWLEDGEMENTS}

J.I. Hidalgo and O. Garnica work has been supported by Fundación Eugenio Rodríguez Pascual 2019 grant, Madrid Regional Government grant B2017/BMD3773 (GenObIA-CM), FEDER funds, and
Madrid Regional Government grant Y2018/NMT-4668 (Micro-StressMAP-CM).

\section{REFERENCES}

[1] Anthony Brabazon and Michael O’Neill. 2004. Evolving technical trading rules for spot foreign-exchange markets using grammatical evolution. Computational Management Science 1, 3 (October 2004), 311-327.

[2] W.L. Clarke, D. Cox, L.A . Gonder-Frederick, W. Carter, and S.L. Pohl. 1987. Evaluating clinical accuracy of systems for self-monitoring of blood glucose. Diabetes Care 10, 5 (1987), 622-628.

[3] J. Manuel Colmenar, José L. Risco-Martín, David Atienza, Oscar Garnica, J. Ignacio Hidalgo, and Juan Lanchares. 2010. Improving reliability of embedded systems through dynamic memory manager optimization using grammatical evolution. In Proceedings of the 12th annual conference on Genetic and evolutionary computation (GECCO '10). ACM, New York, NY, USA, 1227-1234.

[4] Iván Contreras, J Ignacio Hidalgo, and Laura Nuñez-Letamendía. 2017. A hybrid automated trading system based on multi-objective grammatical evolution. Journal of Intelligent \& Fuzzy Systems 32, 3 (2017), 2461-2475.

[5] Andy Field. 2009. Discovering statistics using SPSS. Sage publications.

[6] Internation Diabetes Foundation. 2018. IDF Diabetes Atlas 2017. http:// diabetesatlas.org/. (2018). [Online; accessed 7-November-2018].

[7] J. Ignacio Hidalgo, J. Manuel Colmenar, Gabriel Kronberger, Stephan M. Winkler, Oscar Garnica, and Juan Lanchares. 2017. Data Based Prediction of Blood Glucose Concentrations Using Evolutionary Methods. F. Med. Syst. 41, 9 (Sept. 2017), $1-20$.

[8] J. Ignacio Hidalgo, J. Manuel Colmenar, José L. Risco-Martin, Alfredo CuestaInfante, Esther Maqueda, Marta Botella, and José Antonio Rubio. 2014. Modeling glycemia in humans by means of Grammatical Evolution. Applied Soft Computing 20 (2014), 40-53.

[9] Ulya Rahmet Karpuzcu. 2005. Automatic Verilog Code Generation through Grammatical Evolution. In Genetic and Evolutionary Computation Conference (GECCO2005) workshop program, Franz Rothlauf et. al (Ed.)

[10] J. R. Koza. 1992. Genetic Programming. The MIT Press, Cambridge, Massachusetts

[11] Nuno Lourenço, Filipe Assunção, Francisco B Pereira, Ernesto Costa, and Penousal Machado. 2018. Structured grammatical evolution: a dynamic approach. In Handbook of Grammatical Evolution. Springer, 137-161.

[12] Nuno Lourenço, Joaquim Ferrer, Francisco B Pereira, and Ernesto Costa. 2017. A comparative study of different grammar-based genetic programming approaches. In European Conference on Genetic Programming. Springer, 311-325.

[13] Nuno Lourenço, Francisco B Pereira, and Ernesto Costa. 2016. Unveiling the properties of structured grammatical evolution. Genetic Programming and Evolvable Machines 17, 3 (2016), 251-289.

[14] Robert I. McKay, Nguyen Xuan Hoai, Peter Alexander Whigham, Yin Shan, and Michael O’Neill. 2010. Grammar-based Genetic Programming: a survey. Genetic Programming and Evolvable Machines 11, 3 (01 Sep 2010), 365-396.

[15] Miguel Nicolau, Matthew Saunders, Michael O'Neill, Bruce Osborne, and Anthony Brabazon. 2012. Evolving Interpolating Models of Net Ecosystem CO2 Exchange Using Grammatical Evolution. In Genetic Programming, Alberto Moraglio, Sara Silva, Krzysztof Krawiec, Penousal Machado, and Carlos Cotta (Eds.). Lecture Notes in Computer Science, Vol. 7244. Springer Berlin Heidelberg, 134-145.

[16] Michael O'Neill and Conor Ryan. 2001. Grammatical evolution. IEEE Trans. Evolutionary Computation 5, 4 (2001), 349-358.

[17] Conor Ryan, Michael O'Neill, and JJ Collins. 2018. Handbook of Grammatical Evolution. Springer, Springer Nature.

[18] P.A. Whigham. 1995. Grammatically-based Genetic Programming. In Proceedings of the Workshop on Genetic Programming: From Theory to Real- World Applications. Tahoe City, California, USA. 3341. 

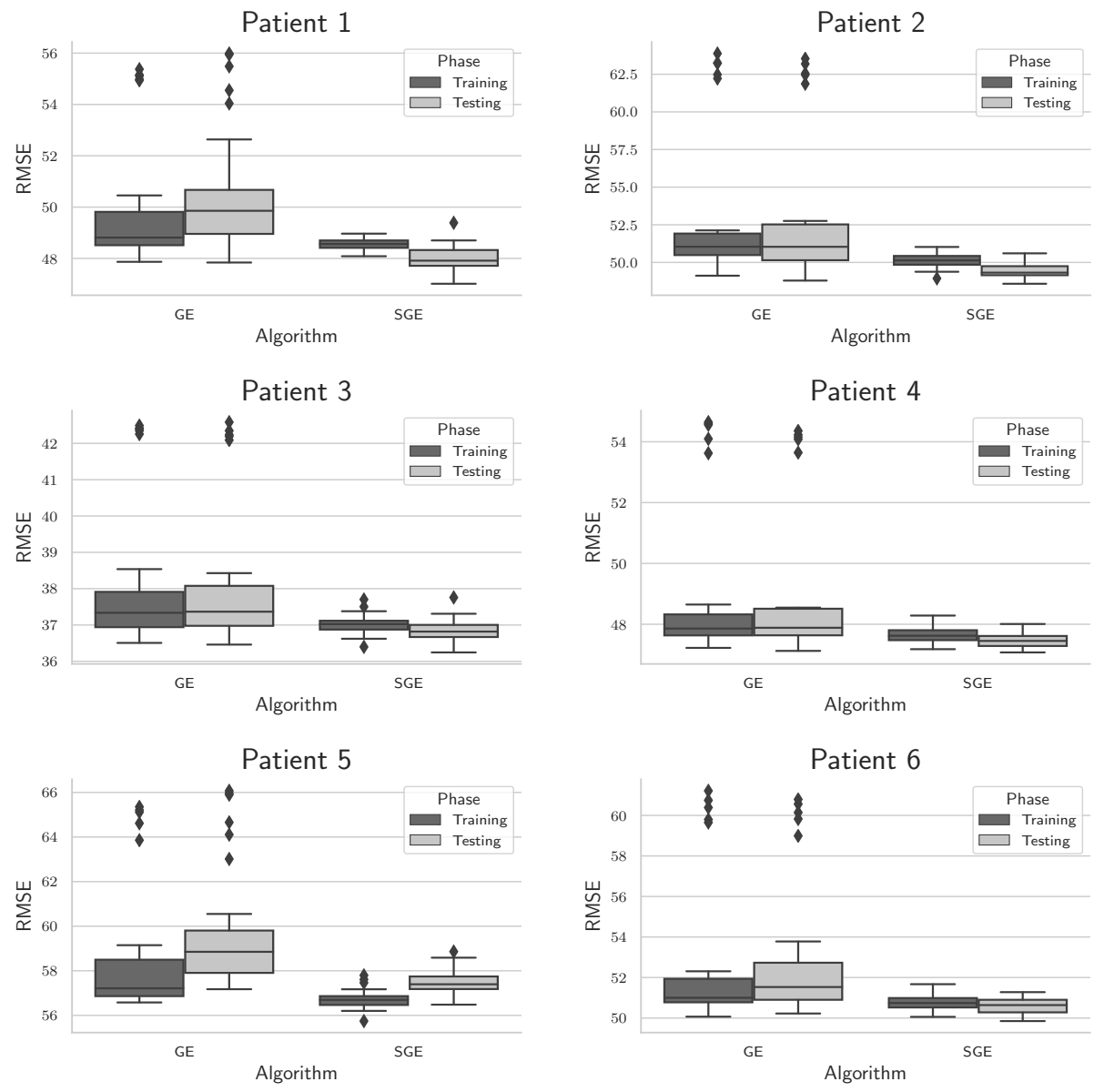

Patient 7
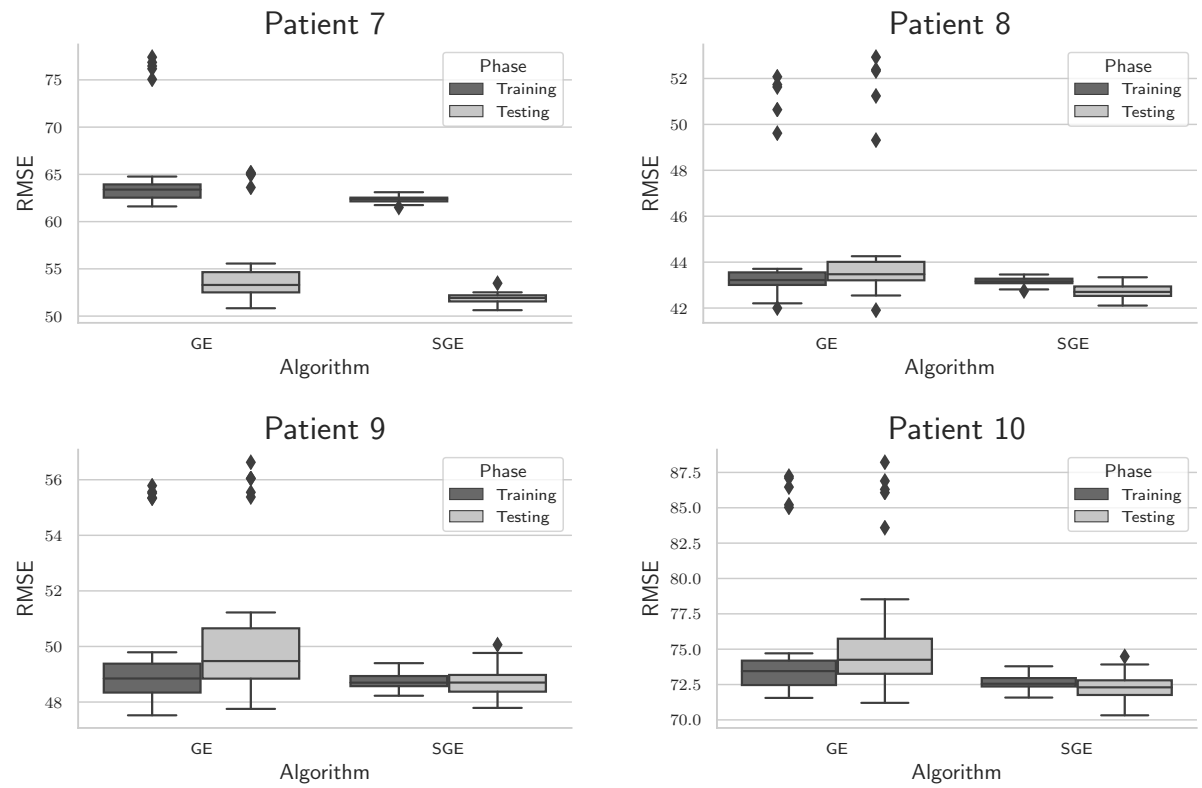

Figure 4: Boxplot with the test results for GE and SGE for the 10 patients considered in this study. Notice the different scale of each plot. 

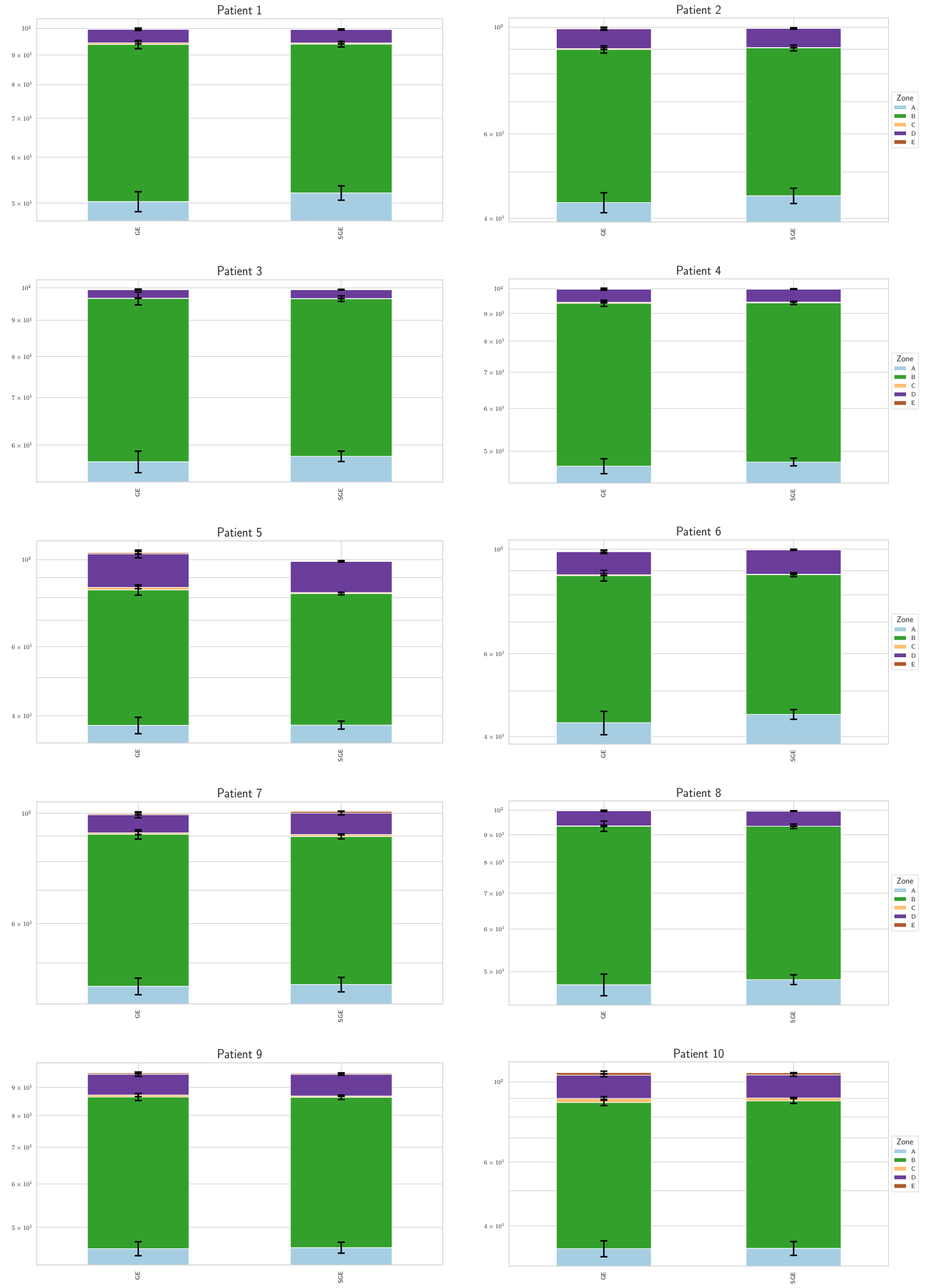

Figure 5: Mean number of points by the zones of Clarke Error Grid for the test data. Each plot represents the percentage of points that are in each zone, for all the patients considered in the study. Results are averages of 30 runs. 